\title{
Self-Assembly of Ordered Microparticle Monolayers from Drying a Droplet on a Liquid Substrate
}

\author{
Weibin Li, ${ }^{\circledR}$ Wenjie Ji, ${ }^{\circledR}$ Ding Lan,** and Yuren Wang* \\ National Microgravity Laboratory, Institute of Mechanics, Chinese Academy of Sciences, 100190 Beijing, China \\ School of Engineering Science, University of Chinese Academy of Sciences, 100049 Beijing, China
}

Supporting Information

ABSTRACT: Drying droplets on solid substrates has always formed a nonuniform and disordered "coffee ring" stain, which has a great negative effect on the application of inject printing and colloidal assembly. We obtain a macrouniform and micro-ordered pattern through evaporation of a colloidal droplet resting on a liquid substrate. The evaporative convection and the capillary forces were responsible for the formation of the ordered structures, which assembled into a monolayer pattern at the liquid-air interface under the action of the weak capillary flow and shrinkage of the triple line. The central bump deposits with disordered particle stacking on the liquid-liquid interface could be attributed to the fast meeting of the descending particles (gravitational sedimentation) and ascending liquid-liquid interface; they would scatter on the ordered monolayer structure and form the final uniform pattern.
$\mathrm{T}$ he evaporation of a sessile droplet containing micro- or nanoparticles is visible everywhere, such as annoying stains in our daily life or printing marks in industrial production. ${ }^{1}$ This process seems simple, but it involves important physicomechanical phenomena such as the wellknown "coffee ring" effect. ${ }^{2}$ This effect, initially revealed by Deegan et al. in 1997, was attributed to the capillary flow caused by uneven evaporation flux when the contact line was pinned. ${ }^{2,3}$ The coffee ring effect is boring in many application fields, such as complex assembly, ${ }^{4}$ crystallization, ${ }^{5}$ and injectprinting. ${ }^{6}$ Therefore, many researchers tried to eliminate the ring-like stain..$^{-12}$

$\mathrm{Hu}$ and Larson found that the Marangoni flow, caused by surface tension gradients, could recirculate particles from the edge to the center of the droplet and finally form centered stains. ${ }^{7}$ Yunker found that the shape of the suspended particles can be used to eliminate the coffee ring effect and produce uniform deposits during evaporation due to the strong interparticle capillary interactions. ${ }^{13} \mathrm{Li}$ et al. found patterns from an evaporating droplet including a concentrated stain, a ring-like deposit, and a combined structure, which depended on the surface activity of solutes and the contact angle hysteresis $(\mathrm{CAH})$ of substrates. Therefore, they acquired the suppression of the coffee ring effect by controlling the CAH. ${ }^{14}$ These above-mentioned methods can effectively avoid the ring-like stains and obtain relatively uniform patterns visually; however, all of these patterns still have a lot of cracks and defects, and the structures are highly disordered under a mesoscopic or microscopic view. It is quite a challenge to obtain both macrouniform and micro-ordered patterns from an evaporating droplet.

Part of the difficulty for the formation of an ordered structure can be attributed to the inevitable defects on solid surfaces, which will lead to defect formation inside of the patterns. Besides, the mismatch between colloidal gel and a solid surface could result in cracks. ${ }^{15}$ Compared to a solid substrate, the liquid substrate is deformable and flexible, which is more proper for particle assembling. In addition, the structures formed on the liquid surface can be easier to transfer. However, as far as we know, there is still no research focus on the pattern formation from an evaporating droplet on a liquid surface.

In this Letter, we deposit a colloidal droplet on an immiscible and denser liquid substrate. We obtain an ordered microparticle monolayer on the liquid-air interface and central bump deposits on the liquid-liquid interface. Here we make direct, real-time and real-space observations of particles assembling and pattern formation to reveal the mechanisms.

Phenyl methyl silicone oil (PMSO) (ShinEtsu KF-54) was adopted as the liquid substrate for the following reasons: (i) it is hydrophobic and immiscible with aqueous suspensions; (ii) its density is $1.07 \mathrm{~g} / \mathrm{cm}^{3}$, which is denser than water; thus, the aqueous drop can float on the liquid surface; (iii) it is transparent, which is easy for optical observation; and (iv) it is stable and nonvolatile. The surface tension of the PMSO and

Received: July 3, 2019

Accepted: September 25, 2019 
pure water is 25 and $72 \mathrm{mN} / \mathrm{m}$ at $25{ }^{\circ} \mathrm{C}$, respectively. The dynamic viscosity of the PMSO is $400 \mathrm{~mm}^{2} / \mathrm{s}$. The silicon oil is filled in a transparent vessel. In our experiment, the spreading parameter is $S=72-(59+25) \mathrm{mN} / \mathrm{m}=-12 \mathrm{mN} / \mathrm{m}<0$; thus, a liquid lens can be formed; the side-view image can be seen in Figure 1b. The wetting behavior of droplet floating on

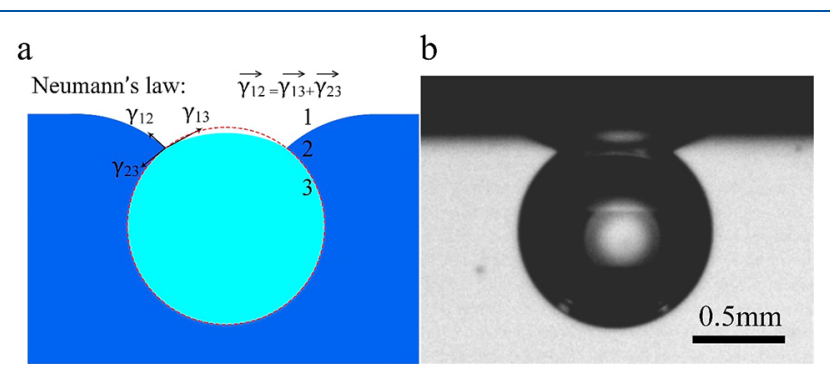

Figure 1. (a) Schematic of a droplet floating on a liquid substrate, which is governed by Neumann's law $\overrightarrow{\gamma_{12}}=\overrightarrow{\gamma_{13}}+\overrightarrow{\gamma_{23}}$. (b) Side-view image of a colloidal droplet on a phenyl-silicon oil surface.

a liquid substrate can be described by Neumann's law. ${ }^{16}$ On the liquid substrate, the three interfaces arrange so that surface tensions balance at the triple line, as shown in Figure 1a; the droplet is made of two spherical caps that satisfy Neumann's vector relation all along the triple line $\overrightarrow{\gamma_{12}}=\overrightarrow{\gamma_{13}}+\overrightarrow{\gamma_{23}}$.

Polystyrene (PS) colloidal particles, dispersed in pure water, were purchased from Duke Scientific Corporation and measured $3 \mu \mathrm{m}$ in diameter. The original mass concentration was $10 \mathrm{wt} \%$, and the nominal density $\rho_{\mathrm{s}}$ was $1.05 \mathrm{~g} / \mathrm{cm}^{3}$. The monodisperse PS colloidal solution used in the experiment was diluted to 0.1 wt \% with high-purity water. Prior to each experiment, the suspensions were stabilized using an ultrasonic cleaner (KQ2200DE, Kunshan Ultrasonic Instruments) for at least $10 \mathrm{~min}$.

A colloidal droplet with a volume of $0.5 \mu \mathrm{L}$ was injected through an electronic syringe unit (high precision, fully automatic electric injection) on the liquid substrate, and the droplet shape was captured by a CCD camera from a side view (Dataphysics, OCA15EC). The final deposition patterns were recorded using a microscope equipped with a digital camera (A1R-si, Nikon) from a bottom view. All experimental apparatus were placed on an optical platform to avoid fluctuation of the liquid level. Images were analyzed in IMAGE-PRO PLUS 6.0 software. Experiments were repeated three times to establish reproducibility. All experiments were performed at an approximate environmental temperature of 22 ${ }^{\circ} \mathrm{C}$ and $23 \%$ relative humidity.

The colloidal particles are always assembling by relying on the interfaces. For an evaporating droplet resting on a solid substrate, the researcher cared about the interfacial phenomena, such as particle accumulation at the triple line, the assembly process at the liquid-air interface, and capillary interactions at the liquid-solid interface. The assembling behaviors at these interfaces determined the final deposition patterns all together. Similarly, considering a droplet floating on a liquid substrate, it can be speculated that the crucial regions of the droplet for particles self-assembling include the triple line, the liquid-air interface, and the liquid-liquid interface. Therefore, the particle dynamics at these three locations were observed and studied.

To illustrate the contact line behavior of both aqueous and colloidal droplet on a liquid surface, we draw the curves of the contact radius tatio $\left(R / R_{\mathrm{i}}\right)$ to the evaporation time ratio $\left(t / t_{\mathrm{f}}\right)$, where $R / R_{\mathrm{i}}$ is the ratio of the contact radius to the initial contact radius and $t / t_{\mathrm{f}}$ is the ratio of the evaporating time to the total evaporation time. The red and blue dotted lines represent aqueous and colloidal droplet, respectively. There is no irregularity or defect on the liquid surface; therefore, the triple line will slip easily as with evaporation, which is different from the pinning effect of a solid surface (see the red dotted line in Figure $2 \mathrm{f}$ ). The triple line behavior of the colloidal
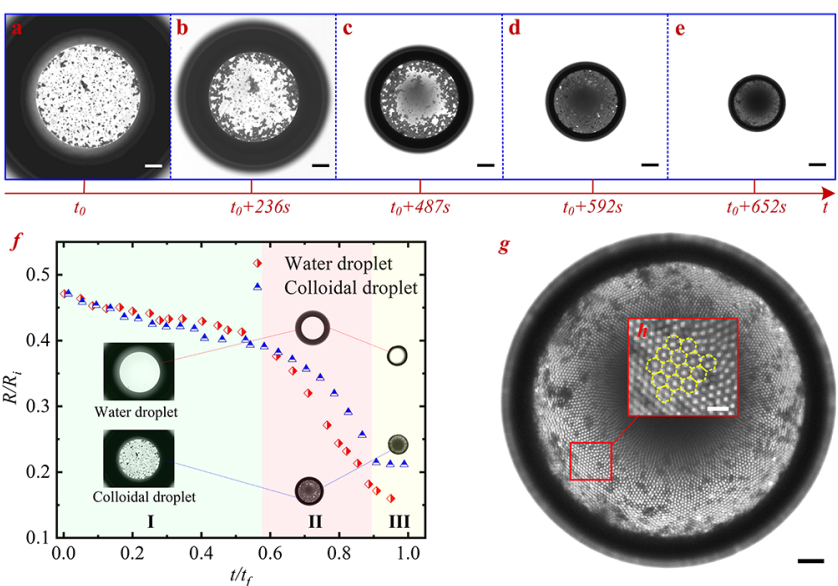

Figure 2. (a-e) Self-assembly of particles at the liquid-air interface as the droplet evaporates, where the inner perimeter represents the triple line. The scale bar is $70 \mu \mathrm{m}$. (f) Change of the contact radius ratio with the evaporation time ratio; the red and blue lines represent water droplet and colloidal droplet (PS; particle radius: $3 \mu \mathrm{m}$; volume fraction: 0.1 wt \%), respectively. (g) Microscope image under magnification of (e); the scale bar is $20 \mu \mathrm{m}$. (h) Enlarged closepacked structure in the red rectangular region of $(\mathrm{g})$; the scale bar is 6 $\mu \mathrm{m}$.

droplet is different from that of water because particles confined at a contact line have the self-pinning effect. ${ }^{17}$ On the basis of the curves of Figure $2 \mathrm{f}$, the evaporation process can be divided into three stages. In stage I, these two curves almost coincide with each other, and the contact radius decreases slowly, which means that the dispersive particles have little resistance to the triple line (Figure 2a). In stage II, the triple line of the water droplet shrinks faster than that of the colloidal droplet because these particles and islands aggregate near the triple line and hinder the motion of the contact line. In the first two stages, particles near the triple line can be pushed by the contact line and aggregate together due to the small friction resistance between particles and the liquid substrate (Figure $2 b-d)$. In stage III, the contact radius for the water droplet decreases continuously, while the contact line of the colloidal droplet remains pinned. This is because the particles and islands compact each other and assemble into a monolayer (Figure 2e), which prevents shrinkage of the triple line.

According to Deegan et al., ${ }^{2}$ the coffee ring would form from an evaporating droplet containing solutes or particles under certain conditions, such as the pinning triple line and the nonuniform evaporation flux. In this experimental system, particles or ordered islands would be carried toward the contact line and aggregated together, as shown in Figure $2 a-c$ (see Movie 1 in the SI, 11X playing speed). The uneven evaporation flux caused by the meniscus of the liquid-air interface is responsible for the interfacial transport of particles, 


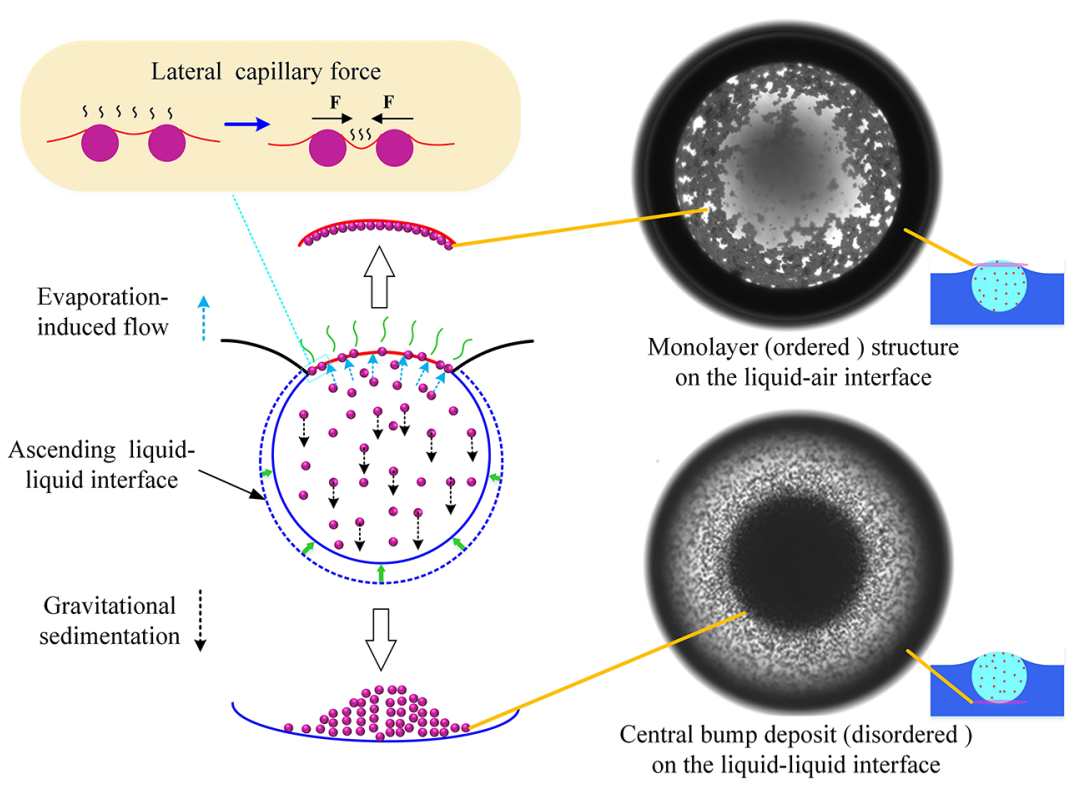

Figure 3. Schematic of the particles assembling on the liquid-air interface and sedimentation on the liquid-liquid interface. Upper left: lateral capillary force between two particles at the liquid-air interface. Upper right: cross-sectional microscopic image at the liquid-air interface (at the triple line level). Lower right: cross-sectional microscopic image at the liquid-liquid interface (at the underside level).

like the sessile droplet on a solid substrate. ${ }^{18}$ The capillary flow is so weak that particles have enough time to arrange and form an ordered structure. ${ }^{19}$ Therefore, the slippage of the contact line and the weak capillary flow made particles and islands assemble into a two-dimensional (2-D) ordered structure near the triple line.

The evaporation kinetics would be likely to affect the dynamic behavior of particles at the liquid-air interface; therefore, particles self-assembling at this interface raised our consideration. The aggregation of particles on a liquid-air interface over time was recorded. First, there existed some dispersed particles and sparse islands on the interface, as shown in Figure 2a. As the evaporation continued, these particles aggregated and the islands grew up; they would further move and combine together with shrinkage of the triple line. Finally, a monolayer structure appeared at the liquid-air interface, as shown in Figure 2e (see Movie 2 in the SI, 13X playing speed). The 2-D microparticle monolayers have a hexagonal closed-packed (hcp) structure, as shown in the yellow hexagon in Figure $2 \mathrm{~h}$.

For an evaporating droplet, there is frequent matter and energy exchange with the environment. Liquid molecules interchange rapidly between the liquid-air interface and the adjacent air, and the evaporated solvent molecules must be replenished from the liquid phase below. Therefore, there must be a net relative flux of solvent molecules toward the interface. In this case, microparticles would be carried by the solvent flow and impinge the interface. These particles would be captured by the liquid-air interface and further assemble into isolated islands. ${ }^{20,21}$ Particle islands were transported toward the triple line under the action of the outward capillary flow and merged into larger islands, which agreed well with the observation results in Figure $2 b, c$. In addition, the shrinkage of the triple line resulted in compression of the assembled islands. During the later stages, homogeneous particle monolayers formed on the liquid substrate.

We assumed that droplet evaporation occurs in a quasisteady and diffusion-limited fashion. Besides, the droplet shape is approximately spherical, and the evaporation flux is uniform along the surface of the droplet. According to Maxwell's studies, the rate of mass loss by evaporation was given as ${ }^{22,23}$

$$
\frac{\mathrm{d} m}{\mathrm{~d} t}=4 \pi R_{0} D_{\mathrm{v}}\left(c_{\mathrm{v}}-c_{\infty}\right)=4 \pi R_{0} D_{\mathrm{v}} c_{\mathrm{v}}(1-H)
$$

where $m$ is the mass, $D_{\mathrm{v}}$ is the diffusion coefficient, $R_{0}$ is the radius of the spherical droplet from the center to the surface, $c_{\infty}$ and $c_{\mathrm{v}}$ are the concentrations of vapor at infinite distance and at its surface (at $R_{0}$ distance), and $H$ is the relative humidity. The uniform evaporation flux $J$ can be expressed as

$$
J=\frac{\mathrm{d} m}{\mathrm{~d} t} / 4 \pi R_{0}^{2}
$$

The solvent velocity induced by evaporation flux can be expressed as ${ }^{24}$

$$
v_{\mathrm{s}}=J / \rho
$$

where $\rho$ is the fluid density. Combining eqs $1-3$, the solvent velocity can be expressed as

$$
v_{\mathrm{s}}=\frac{D_{\mathrm{v}} c_{\mathrm{v}}(1-H)}{\rho R_{0}}
$$

The Brownian motion of a particle was described by Einstein's theory, and the mean square displacement (MSD) $[\Delta x(t)]^{2}$ in one dimension of a free Brownian particle during time $t$ is expressed as ${ }^{25}$

$$
\left\langle[\Delta x(t)]^{2}\right\rangle=2 D t
$$

where $D$ is the diffusion constant. The mean Brownian velocity of particles measured over an interval of time $t$ is ${ }^{26}$

$$
\bar{v}_{\mathrm{b}} \equiv \sqrt{\left\langle[\Delta x(t)]^{2}\right\rangle} / t=\sqrt{2 D} / \sqrt{t}
$$

The diffusion constant $D$ can be estimated from the StokesEinstein relation $D=k_{\mathrm{B}} T / 6 \pi \eta r,{ }^{27}$ where $k_{\mathrm{B}}$ is the Boltzmann constant, $T$ is the temperature, $\eta$ is the viscosity of water, and $r$ is the hydrodynamic radius of the particle that approximates to 
the particle radius. In our experimental system, $D_{\mathrm{v}}=25.3$ $\mathrm{mm}^{2} / \mathrm{s}, c_{\mathrm{v}}=2 \times 10^{-5} \mathrm{~g} / \mathrm{cm}^{3}, H=23 \%, \rho=1 \mathrm{~g} / \mathrm{cm}^{3}, R_{0}=0.49$ $\mathrm{mm}, k_{\mathrm{B}}=1.38 \times 10^{-23} \mathrm{~J} / \mathrm{K}, T=295.15 \mathrm{~K}, \eta=0.9579 \times 10^{-3}$ $\mathrm{N} \cdot \mathrm{s} / \mathrm{m}^{2}$, and $r=1.5 \mu \mathrm{m}$. Here we consider the competitive relations of the solvent velocity and Brownian motion velocity in local regions near the liquid-air interface; therefore, the particle size can be chosen as the characteristic length. The characteristic time can be calculated as $t=2 r / v_{s}$, and the mean Brownian velocity can be estimated as $\bar{\nu}_{\mathrm{b}}=0.28 \mu \mathrm{m} / \mathrm{s}$, which is less than the solvent velocity $v_{\mathrm{s}}=0.8 \mu \mathrm{m} / \mathrm{s}$. The solvent velocity is obviously fast compared to diffusion; therefore, the particles can be easily captured by the liquid-air interface. It is worth mentioning that the thermal Marangoni effect was weak in this experimental system. ${ }^{28}$ Thus, the evaporative convection dominated the particle dynamics at the liquid-air interface.

These captured particles would finally assemble and form ordered monolayers, as shown in Figure 2e,g. The observed attraction and coalescence of particle aggregates can be attributed to the action of the lateral capillary forces; ${ }^{29}$ this interaction is due to the overlap of interfacial deformations created by the wetting properties of the particle surface (see the upper left of Figure 3). The results from the liquid-air interface confirm that the evaporation-induced convection and the capillary forces are the main factors governing the 2-D array formation.

The particle dynamic behaviors at the liquid-air and liquid-liquid interfaces seem different, as shown in Figure 3. The lower right in Figure 3 shows the micrograph at the underside level, i.e., the liquid-liquid interface. It can be observed that a lot of particles deposit and rest on the liquidliquid interface unorderly. The particles would accumulate and deposit on liquid substrate over time, which could be attributed to the fast meeting of the gravitational sedimentation of particles and ascending liquid-liquid interface. ${ }^{30}$ For an evaporating droplet, the terminal velocity of a particle falling in a fluid can be described by the Stokes law ${ }^{31}$

$$
v_{\mathrm{p}}=\frac{2 r^{2} \Delta \rho g}{9 \eta}
$$

where $v_{\mathrm{p}}$ is the terminal velocity, $g$ is the gravitational constant, and $\Delta \rho$ is the difference in density of the particle $\left(\rho_{\mathrm{s}}\right)$ and dispersed phase $(\rho)$. With an increase of the particle size, the sedimentation rate increases, and the particles would settle faster. The upward movement of the liquid-liquid interface due to evaporation could be expressed as $v_{\mathrm{e}}=2 R_{0} / t_{\mathrm{f}}$. From our experiments, $\Delta \rho=\rho_{\mathrm{s}}-\rho=50 \mathrm{~kg} / \mathrm{m}^{3}, g=10 \mathrm{~m} / \mathrm{s}^{2}$, and $t_{\mathrm{f}}=$ $724 \mathrm{~s}$, Thus, the terminal velocity is $v_{\mathrm{p}}=0.26 \mu \mathrm{m} / \mathrm{s}$, and the ascending velocity of the liquid-liquid interface is $v_{\mathrm{e}}=1.35$ $\mu \mathrm{m} / \mathrm{s}$. The relative velocity of the sedimentation of particles and the ascending interface is $v_{\mathrm{e}}+v_{\mathrm{p}}=1.61 \mu \mathrm{m} / \mathrm{s}$. Therefore, the falling particles would be captured by the ascending liquid-liquid interface rapidly. There is not enough time for these particles to find an appropriate spot and form an ordered structure. ${ }^{19}$ Therefore, the particles would form a disordered phase on the liquid-liquid interface, as shown in the lower right of Figure 3. In addition, unlike the radial solvent flow caused by evaporation, the direction of the particle sedimentation is downward, which makes more particles concentrate near the apex of the droplet and finally form a central bump deposit at a macroscale (red circle in Figure 4a).
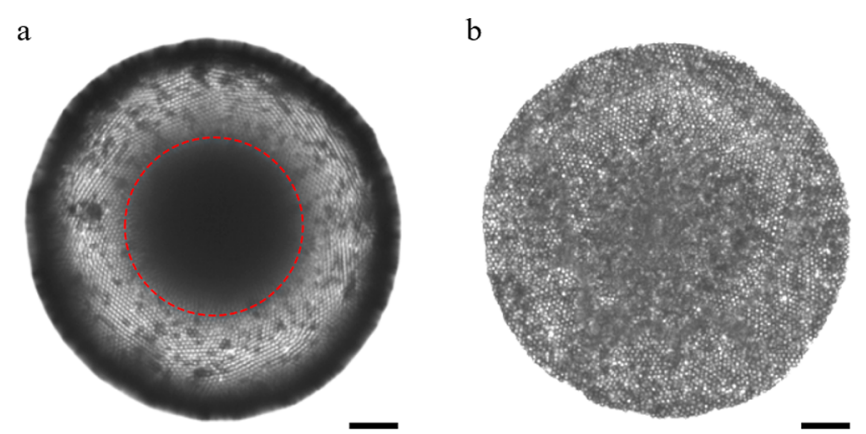

Figure 4. (a) Rearrangement of particles from the sediment at the liquid-liquid interface on the 2-D ordered structure at the liquid-air interface in the final stage of evaporation; the shadow region in the red circle is particle sedimentation. (b) Final deposition patterns on the liquid substrate. The scale bar is $30 \mu \mathrm{m}$.

As with evaporation, the liquid-liquid interface was gradually getting close to the liquid-air interface. In the final stage, the droplet evolved into a thin aqueous liquid film, which made the particles at liquid-air and liquid-liquid interfaces stack up. Therefore, those ordered and disordered structure would rearrange and form new patterns (Figure 4). As shown in Figure 4a, the particles located at the liquid-air interface had already assemble into an ordered monolayer, which had a hcp crystal structure with a certain strength; therefore, the colloidal crystal structure at the liquid-air interface ceased to reassemble. However, for the central bump deposits containing loosely stacked particles at the liquidliquid interface, those particles would scatter under the action of surface tension and rearrange based on the ordered monolayer, as shown in Figure $4 \mathrm{~b}$. Therefore, the final pattern included an ordered monolayer with above disordered particles stacking.

In conclusion, we have studied an evaporating colloidal droplet resting on a liquid substrate. It is shown that the final deposit patterns are determined by a combination of different physical effects at the contact line, the liquid-air interface, and the liquid-liquid interface. We demonstrate that three key elements are required for the interfacial self-assembly mechanism: (i) evaporation, to make particles near the liquid-air interface be captured by the interface; (ii) an attractive interaction between these particles, to prompt particle aggregation and form islands; and (iii) shrinkage of the triple line and weak capillary flow, to piece small islands together and form a monolayer structure. The central bump deposits with disordered particle stacking on the liquid-liquid interface could be attributed to the fast meeting of the gravitational sedimentation of particles and ascending liquidliquid interface; they would scatter on the ordered monolayer and form the final uniform pattern.

\section{ASSOCIATED CONTENT}

\section{S Supporting Information}

The Supporting Information is available free of charge on the ACS Publications website at DOI: 10.1021/acs.jpclett.9b01917.

Video showing the formation of ordered structures near the triple line: motion of particles and islands toward the contact line under the capillary flow (MP4)

Video showing the formation of ordered structures at the liquid-air interface: particles and islands assemble 
into an ordered monolayer structure under the action of the slippage of the contact line, the capillary flow, and the evaporation convection (MP4)

\section{AUTHOR INFORMATION}

\section{Corresponding Authors}

*E-mail: landing@imech.ac.cn.

*E-mail: yurenwang@imech.ac.cn.

ORCID

Weibin Li: 0000-0002-6406-1553

Wenjie Ji: 0000-0002-6560-0731

Notes

The authors declare no competing financial interest.

\section{ACKNOWLEDGMENTS}

The authors gratefully acknowledge financial support from National Natural Science Foundation of China (Grant No. 11902321 and U1738118) and the Strategic Priority Research Program of the Chinese Academy of Sciences (Grant No. XDB22040301).

\section{REFERENCES}

(1) Zang, D.; Tarafdar, S.; Tarasevich, Y. Y.; Dutta Choudhury, M.; Dutta, T. Evaporation of a Droplet: From physics to applications. Phys. Rep. 2019, 804, 1-56.

(2) Deegan, R. D.; Bakajin, O.; Dupont, T. F.; Huber, G.; Nagel, S. R.; Witten, T. A. Capillary flow as the cause of ring stains from dried liquid drops. Nature 1997, 389, 827-829.

(3) Deegan, R. D.; Bakajin, O.; Dupont, T. F.; Huber, G.; Nagel, S. R.; Witten, T. A. Contact line deposits in an evaporating drop. Phys. Rev. E: Stat. Phys., Plasmas, Fluids, Relat. Interdiscip. Top. 2000, 62, $756-765$.

(4) Han, W.; Lin, Z. Learning from "Coffee Rings": Ordered Structures Enabled by Controlled Evaporative Self-Assembly. Angew. Chem., Int. Ed. 2012, 51, 1534-1546.

(5) Han, M.; Li, J.; He, G.; Lin, M.; Xiao, W.; Li, X.; Wu, X.; Jiang, $\mathrm{X}$. Tailored $3 \mathrm{D}$ printed micro-crystallization chip for versatile and high-efficiency droplet evaporative crystallization. Lab Chip 2019, 19, 767-777.

(6) Liu, H.; Xu, W.; Tan, W.; Zhu, X.; Wang, J.; Peng, J.; Cao, Y. Line printing solution-processable small molecules with uniform surface profile via ink-jet printer. J. Colloid Interface Sci. 2016, 465, 106-111.

(7) Hu, H.; Larson, R. G. Marangoni effect reverses coffee-ring depositions. J. Phys. Chem. B 2006, 110, 7090-7094.

(8) Das, S.; Dey, A.; Reddy, G.; Sarma, D. D. Suppression of the coffee-ring effect and evaporation-driven disorder to order transition in colloidal droplets. J. Phys. Chem. Lett. 2017, 8, 4704-4709.

(9) Wray, A. W.; Papageorgiou, D. T.; Craster, R. V.; Sefiane, K.; Matar, O. K. Electrostatic suppression of the "coffee stain effect. Langmuir 2014, 30, 5849-5858.

(10) Sun, P.; Ma, R.; Wang, K.; Zhong, M.; Wei, J.; Wu, D.; Sasaki, T.; Zhu, H. Suppression of the coffee-ring effect by self-assembling graphene oxide and monolayer titania. Nanotechnology 2013, 24, 075601.

(11) Cui, L.; Zhang, J.; Zhang, X.; Huang, L.; Wang, Z.; Li, Y.; Gao, H.; Zhu, S.; Wang, T.; Yang, B. Suppression of the coffee ring effect by hydrosoluble polymer additives. ACS Appl. Mater. Interfaces 2012, 4, 2775-2780.

(12) Mampallil, D.; Reboud, J.; Wilson, R.; Wylie, D.; Klug, D. R.; Cooper, J. M. Acoustic suppression of the coffee-ring effect. Soft Matter 2015, 11, 7207-7213.

(13) Yunker, P. J.; Still, T.; Lohr, M. A.; Yodh, A. G. Suppression of the coffee-ring effect by shape-dependent capillary interactions. Nature 2011, 476, 308-311.
(14) Li, Y.-F.; Sheng, Y.-J.; Tsao, H.-K. Evaporation stains: suppressing the coffee-ring effect by contact angle hysteresis. Langmuir 2013, 29, 7802-7811.

(15) Kim, J. Y.; Cho, K.; Ryu, S.-a.; Kim, S. Y.; Weon, B. M. Crack formation and prevention in colloidal drops. Sci. Rep. 2015, 5, 13166.

(16) De Gennes, P.-G.; Brochard-Wyart, F.; Quéré, D. Capillarity and wetting phenomena: drops, bubbles, pearls, waves; Springer Science \& Business Media, 2013.

(17) Weon, B. M.; Je, J. H. Self-pinning by colloids confined at a contact line. Phys. Rev. Lett. 2013, 110, 028303.

(18) Gleason, K.; Putnam, S. A. Microdroplet evaporation with a forced pinned contact line. Langmuir 2014, 30, 10548-10555.

(19) Marín, A. G.; Gelderblom, H.; Lohse, D.; Snoeijer, J. H. Order to Disorder Transition in Ring-Shaped Colloidal Stains. Phys. Rev. Lett. 2011, 107, 085502.

(20) Li, Y.; Yang, Q.; Li, M.; Song, Y. Rate-dependent interface capture beyond the coffee-ring effect. Sci. Rep. 2016, 6, 24628.

(21) Bigioni, T. P.; Lin, X.-M.; Nguyen, T. T.; Corwin, E. I.; Witten, T. A.; Jaeger, H. M. Kinetically driven self assembly of highly ordered nanoparticle monolayers. Nat. Mater. 2006, 5, 265-270.

(22) Maxwell, J. C. Collected Scientific Papers; Cambridge, 1890; Vol. 2, p 625 .

(23) Erbil, H. Y. Evaporation of pure liquid sessile and spherical suspended drops: A review. Adv. Colloid Interface Sci. 2012, 170, 6786.

(24) Hu, H.; Larson, R. G. Analysis of the Microfluid Flow in an Evaporating Sessile Droplet. Langmuir 2005, 21, 3963-3971.

(25) Einstein, A. Investigations on the Theory of the Brownian Movement; Dover Publications: New York, 1956.

(26) Li, T. C.; Kheifets, S.; Medellin, D.; Raizen, M. G. Measurement of the Instantaneous Velocity of a Brownian Particle. Science 2010, 328, 1673-1675.

(27) Jones, R. A. Soft condensed matter; Oxford University Press, 2002.

(28) Hu, H.; Larson, R. G. Analysis of the effects of Marangoni stresses on the microflow in an evaporating sessile droplet. Langmuir 2005, 21 (9), 3972-3980

(29) Kralchevsky, P. A.; Denkov, N. D. Capillary forces and structuring in layers of colloid particles. Curr. Opin. Colloid Interface Sci. 2001, 6 (4), 383-401.

(30) Li, W.; Ji, W.; Sun, H.; Lan, D.; Wang, Y. Pattern Formation in Drying Sessile and Pendant Droplet: Interactions of Gravity Settling, Interface Shrinkage, and Capillary Flow. Langmuir 2019, 35 (1), 113119.

(31) Stokes, G. G. Mathematical and Physical Papers; Cambridge, 1901. 